\title{
La mission médicale de Louis-André Gosse pendant son séjour en Grèce (1827-1829)
}

par Michelle Bouvier-Bron

\section{Résumé}

Louis-André Gosse (1791-1873), médecin genevois de tendance libérale, ému par la misère qui sévissait en Grèce au cours de la guerre d'indépendance, partit se mettre au service du jeune Etat et y séjourna de 1827 à 1829. A côté de tâches administratives - celle de commissaire de la flotte, puis de collecteur des impôts dans l'Archipel - il prend une part active, dès le printemps 1828, à la demande du président de la Grèce, Jean Capodistrias, dans la lutte contre l'épidémie de peste qui éclate à ce moment-là. Cette maladie, depuis longtemps disparue de Grèce, y a été ramenée par l'armée égyptienne et se répand à Egine, Hydra, Spetsès et dans les provinces d'Argolide, de Mégaride et d'Achaïe. Elle sera jugulée grâce aux mesures draconiennes prises à temps et appliquées rigoureusement, mais fera tout de même plusieurs centaines de victimes. Lous-André Gosse, qui a travaillé sur le terrain en Mégaride en juin 1828, puis en Achaïe en janvier 1829, a laissé un récit détaillé de cette expérience et expose ses idées sur la maladie, son traitement et les mesures préventives.

Louis-André Gosse (1791-1873) est une des figures marquantes de la médecine sociale genevoise du XIX ${ }^{e}$ siècle. Fils unique du pharmacien HenriAlbert Gosse, l'un des fondateurs de la Société helvétique des sciences naturelles, Louis-André Gosse fit ses humanités et des études de sciences naturelles à Genève. Après un stage dans la pharmacie de son père, il part étudier la médecine à Paris où il obtient son diplôme en 1816. Puis il entreprend, pendant quatre ans, une sorte de tour d'Europe, séjournant en Italie, Autriche, Hollande, Angleterre et Irlande.

En 1820, il revient à Genève, où il fonde avec les $\mathrm{D}^{\text {rs }}$ Jean-Louis Prevost et Dupin un Dispensaire des médecins, sur un modèle anglais. Militant dans le parti libéral, il siège de 1822 jusqu'en 1834, exception faite des années passées en Grèce, au Conseil représentatif; il y proposera deux mesures importantes: la suppression du carcan et de l'exposition publique des 
criminels, et l'abolition des mesures antisémites datant de 1816. Il est également l'un des fondateurs du «Journal de Genève», dont le premier numéro paraît le 5 janvier 1826. Gosse y signe de nombreux articles sur des questions d'hygiène publique et de médecine.

Dans son ensemble, le «Journal de Genève» s'intéressait avant tout aux questions locales et confédérales; mais y figuraient aussi quelques nouvelles de l'étranger. Proportionnellement, ce sont, de loin, les nouvelles concernant la Grèce insurgée - et en 1826, celles dépeignant le sort des assiégés de Missolonghi - qui y tiennent le plus de place. Ce qui s'explique par le fait qu'après l'anéantissement des révolutions libérales de Naples, du Piémont et de l'Espagne, la Grèce est le dernier bastion du combat pour la liberté, que la Sainte-Alliance ne peut pas contrer avec autant de vigueur que les autres, puisqu'il s'agit également d'une lutte de chrétiens contre des musulmans.

Pour situer brièvement le contexte historique dans lequel Louis-André Gosse évoluera, rappelons que l'étendard de l'insurrection de la Grèce a été levé le 25 mars 1821 par l'archevêque de Patras, Germanos, au couvent d'Agia Lavra près de Kalavryta. Le sultan, dont les troupes étaient retenues par la rébellion d'Ali de Tébélen, pacha de Janina, s'est trouvé surpris par l'ampleur du mouvement, si bien qu'au cours de l'année 1821, les Grecs se rendent maîtres du Péloponnèse, qu'on appelle à l'époque la Morée, et d'une bonne partie des îles; ils peuvent ainsi proclamer l'indépendance de la nation à Epidaure le 13 janvier 1822 .

Au cours des années 1823-1824, la situation sur le terrain reste à l'avantage des Grecs. Elle se retourne contre eux lorsque le sultan fait appel à l'un de ses grands vassaux, Méhémet Ali, pacha d'Egypte, dont le fils Ibrahim débarque à Modon en février 1825 et s'établit solidement à Navarin. Pendant deux ans, il ravage le Péloponnèse, alors que l'armée turque est massée en Grèce continentale. Ibrahim ira lui prêter main forte lors de l'interminable siège de la place de Missolonghi (25 avril 1825-22 avril 1826). La mort hérö̈que des défenseurs, le sort des femmes et des enfants survivants vendus comme esclaves, eurent un retentissement extraordinaire en Europe, marquant le point culminant du philhellénisme et entraînant pour une bonne part l'intervention des gouvernements français, anglais et russe. Ils signent le Traité de Londres le 6 juillet 1827, et leurs escadres anéantiront à Navarin la flotte turco-égyptienne le 20 octobre de la même année.

Mais revenons à notre héros! Un jour où Gosse était allé chercher des nouvelles sur la Grèce pour le «Journal de Genève» chez le célèbre philhellène 
genevois Jean Gabriel Eynard, celui-ci lui remet une lettre de la veuve de Marcos Botzaris, l'un des héros et victimes du siège.

Gosse en est bouleversé :

«En sortant de la maison de M. Eynard, je me mis à lire cette lettre dont les détails étaient navrants, et je n'étais pas arrivé à la maison du Calabri [le bastion Mi-Rond, à quelques mètres du Palais Eynard], sans éprouver un sentiment de compassion si fort que je rentrai chez M. Eynard, pour m'offrir à partir pour la Grèce, et d'y porter des secours médicaux. Cette résolution était peut-être bien hasardée, car je n'avais consulté ni mes intérêts propres, étant à la tête d'une nombreuse clientèle médicale, ni l'assentiment de ma bonne mère, dont j'étais le fils unique. ${ }^{1}$

Eynard, sur le moment, tempère son enthousiasme mais prend note de son offre. Un mois plus tard, en décembre 1826, il le fait appeler pour lui confier une mission officielle fort délicate: les comités philhelléniques avaient rassemblé des fonds considérables qu'ils destinaient à la flotte grecque sous forme de numéraire, de munitions et de vivres, en particulier de farine. Mais vu l'état d'anarchie qui règne en Grèce, ils veulent confier le tout en mains sûres, soit celles de Gosse pour les comités suisses, du docteur Bailly pour le comité de Paris et du colonel Heideck pour la Bavière. Ces mêmes comités souhaitent la nomination de lord Cochrane comme commandant de la flotte grecque, choix ratifié par l'Assemblée nationale de Trézène devant laquelle Cochrane prête serment en mars 1822, et Gosse deviendra ipso facto son bras droit; mais la nomination quasi forcée d'un amiral étranger dans un pays d'excellents et illustres marins - les Canaris, Miaoulis, Tombazis s'étaient imposés au cours de plusieurs années de guerre déjà - était des plus discutables et plaçait notre Genevois dans une situation délicate.

Avant son départ, Gosse fait la connaissance et de Cochrane, de passage à Genève, et surtout du comte Jean Capodistrias, le futur président de la Grèce, qui le charge de rassembler des informations sur le caractère, les faits et les gestes des hommes politiques et des militaires grecs, «un véritable livre noir», nous dit Gosse.

Parti vers le 20 décembre par Chambéry, il passe le Mont-Cenis au clair de lune en traîneau, puis par Turin et Parme gagne Ancône. Il s'embarque le 31 décembre pour une traversée houleuse où il souffre pendant plus de quinze jours du mal de mer, aggravé par l'odeur de la fermentation des farines. Le 16 janvier 1828, le bateau enfin relâche à Zante et ce n'est que le 2 février que Gosse débarque à Nauplie, siège du gouvernement où il retrouve le $D^{r}$ Bailly. Mais devant les tensions politiques qui divisent la place et les convoitises que suscitent les richesses qu'il convoie, il préfère rejoindre Hydra, et finalement 
Poros, où il retrouve le colonel Heideck et l'amiral Tombazis avec lesquels il travaillera la main dans la main.

Nous n'aborderons pas, dans cette courte étude, l'activité principale de Gosse, nommé commissaire de la flotte, chargé de distribuer l'aide des comités, puis, lorsque ces secours seront épuisés, nommé collecteur des impôts dans l'Archipel, les îles étant chargées d'entretenir la flotte, par des taxes, impôts ou prêts. Ce qui l'entraînera dans des aventures périlleuses et hautes en couleur, à Chio, Thermia ou Syra, après avoir été le témoin impuissant de la bataille d'Athènes. Et nous concentrerons le reste de notre travail sur son activité accessoire, celle du médecin, dans laquelle il est nettement plus à l'aise que dans les tâches administratives.

Contrairement à ce que nous imaginions - et Gosse de même - la Grèce n'était pas dépourvue de médecins. Dans sa Relation de la peste qui a régné en Grèce en 1827 et $1828^{2}$, Gosse cite le nom de plusieurs d'entre eux qui exercent à Hydra, Poros, Spetses, Nauplie, Argos, Egine. La plupart avaient fait probablement leurs études à l'université de Padoue, ce qui était en tout cas une vieille tradition dans les familles des îles Ioniennes, rattachées pendant des siècles à la République de Venise. C'est donc fortuitement que Gosse aura l'occasion d'exercer sa profession.

Premier exemple: son intervention au cours de la bataille d'Athènes. A la fin d'avril 1827, des troupes de Grecs et de philhellènes avaient été débarquées par la flotte de Cochrane au Pirée et au Phalère - Gosse dit fièrement dans son $S e ́ j o u r{ }^{3}$ : «Je fournis les bâtiments et les vivres pour cette expédition hydriote, spezziote et autres» - elles étaient chargées de débloquer les soldats du colonel Fabvier assiégés dans l'Acropole par les Turcs depuis plusieurs mois. L'expédition se transforme vite en un désastre. Gosse y soigne en vain le général Karaïskaki mortellement blessé et fait avec l'aide d'un jeune chirurgien anglais deux amputations à bord du brick de l'amiral.

Pendant que je faisais ces amputations, on venait d'un autre côté me demander des vivres, et n'ayant point d'encre, j'étais obligé de faire des bons avec le sang de mes patients et des allumettes en guise de plumes. ${ }^{4}$

Autre exemple: quelques mois plus tard, il est appelé à soigner le prince Paul Bonaparte, fils de Lucien, jeune homme de dix-huit ans qui venait de s’engager et qui est grièvement blessé par un coup parti accidentellement de son propre pistolet.

Le Dr Howe, en mon absence, commença à le soigner jusqu'à mon arrivée, mais tout ce que je pus faire fut de diminuer les souffrances qu'il supporta avec le plus grand courage, 
pendant vingt-deux heures. A 7 heures, le lundi matin, il s'écria: Ah, quelle douleur! et mourut dans mes bras à l'instant. ${ }^{5}$

C'est à partir de l'épidémie de peste qui éclate en 1828 que Gosse est appelé à jouer un rôle important dans le domaine médical. Le premier contact qu'il a avec cette maladie presque mythique, il nous le raconte dans son Séjour ${ }^{6}$ :

Je continuai de résider à Poros jusqu'au moment où l'on vint m'annoncer qu'un Hydriote malade me demandait du secours comme médecin; c'était dans la maison de M. Tombazis. A la porte, je fus accosté par Mavrocordatos [notable originaire de Constantinople] qui me dit à l'oreille: Je vous avertis que c'est un cas de peste et je vous conseille de vous huiler les mains. D'abord fort étonné de cette annonce, j'éprouvai un sentiment assez pénible mais me rassurant promptement, j'entrai auprès du malade comme un soldat monte à l'assaut. C'était la première fois que je voyais un pestiféré. Je lui tâtai le pouls, lui prescrivis quelques remèdes qui ne lui furent pas d'une grande utilité, car quelques heures après il était mort.

Gosse a pris le soin de rédiger un récit détaillé de cette expérience dans sa Relation de la peste qui a régné en Grèce en 1827 et 1828, contenant des vues nouvelles sur la marche et le traitement de cette maladie. La première partie de l'ouvrage est un historique de la propagation de l'épidémie, où Gosse tient à prouver le principe de la contagion que certains de ses collègues récusaient. Cet historique repose sur une documentation très précieuse et étendue, mise à sa disposition par Capodistrias, soit des lettres de médecins aux autorités tant égyptiennes (prises sur des bateaux arraisonnés) que grecques. Il peut ainsi retracer le cheminement de la maladie de façon très précise.

La peste bubonique avait disparu de Grèce depuis des décennies, sinon des siècles, malgré le contact fréquent que les navigateurs grecs entretenaient avec l'Asie mineure, le Moyen-Orient et l'Egypte, où la maladie sévissait à l'état endémique. Le fléau est alors importé par l'armée d'Ibrahim, d'Egypte en Morée. Les médecins français et italiens qui étaient incorporés dans les troupes de santé de cette armée, ont tardé à diagnostiquer la maladie, l'épidémie étant d'ailleurs freinée par les conditions climatiques de l'hiver 1827-1828.

Capodistrias, arrivé en Grèce au début de l'année 1828, a proposé dans un but humanitaire à la mi-mars un échange de prisonniers arabes détenus à Poros contre un certain nombre de familles grecques réduites à l'esclavage à Navarin. L'affaire conclue, l'opération est confiée au major autrichien Prokesch sur le bateau le Veneto, escortant la goélette hydriote l'Aphrodite du capitaine Alexandre Kiossé. Les marins hydriotes, à l'escale, profitèrent de cette circonstance pour renouer avec les Turcs, nous dit Gosse; ils mangèrent avec eux et le capitaine Kiossé leur acheta même un châle pour sa sœur. 


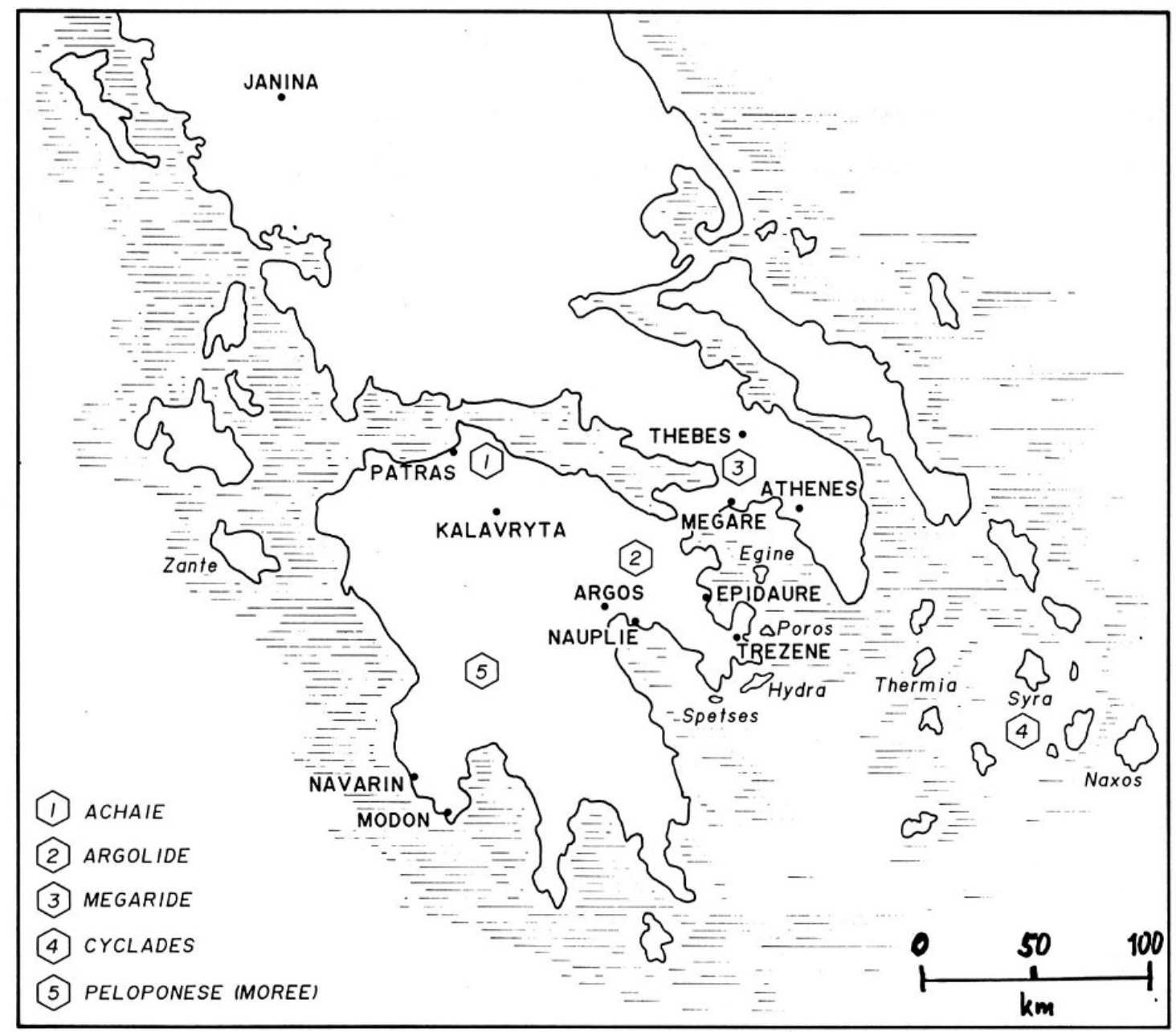

Fig. 1 La Grèce-lieux mentonnés dans le texte

Pendant le voyage de retour, trois hommes de l'équipage, dans chacun des bateaux, meurent. Arrivés à Egine, les passagers sont imprudemment débarqués dans la rade de Perivolia, à une demi-heure du chef-lieu, sans que les autorités sanitaires soient prévenues. Les habitants les reçoivent chez eux et la maladie ne tarde pas à se répandre.

Quant aux marins de l'Aphrodite, ils rentrent à Hydra en passant par Poros. Alexandre Kiossé, après avoir dormi chez son cousin Nicolas, employé par Gosse et ses collègues dans leurs bureaux de marine - c'est son premier malade dont il nous entretenait dans le fragment du Séjour-regagne Hydra, où il tombe immédiatement malade et meurt deux jours plus tard. Bien que le $\mathrm{D}^{\mathrm{r}}$ Xanthos qui l'a soigné ait identifié la maladie, les autorités ne le crurent 
pas et donnèrent la permission d'ensevelir le mort selon les cérémonies habituelles, les proches défilant devant le cercueil ouvert et baisant le front du défunt. La mère et la sœur du défunt, qui en plus avaient ouvert sa malle et trouvé le châle, sont les premières atteintes; mais presque toute la famille sera touchée.

Le sort des marins de l'Aphrodite est tout aussi tragique:

La goëlette Aphrodite, privée de son capitaine et repoussée de toutes les côtes, erra longtemps dans le détroit qui sépare Hydra du continent; elle y fut rencontrée le 22 avril (4 mai) par M. Bétant, mon compatriote, alors secrétaire du Président, et l'équipage lui annonça qu'il avait déjà perdu plusieurs matelots; enfin on eut pitié de sa détresse, et par l'intervention du gouvernement elle fut admise en quarantaine à Spetzia. ${ }^{7}$

Dans cette île, la quarantaine fut organisée de façon tout à fait efficace par le gouverneur, M.Colettis, le futur diplomate, auteur d'ordonnances modèles du genre, qui, appliquées avec rigueur, donnèrent des résultats remarquables.

Gosse, dans son ouvrage, réussit à établir de façon très précise le cheminement du virus, gagnant de Spetses l'Argolide (Merbaka et Chéli et d'autres villages), puis Argos où le $\mathrm{D}^{\mathrm{r}}$ Apostolidis obtint un excellent pourcentage de guérison en hospitalisant ses malades dans la mosquée désaffectée, aux cours vastes et aérées; enfin, la maladie toucha Proïna, le faubourg de Nauplie où s'étaient entassés 2500 réfugiés vivant dans la misère; alors que les mesures rigoureuses prises par Kalerdgi et le colonel Heideck furent assez efficaces pour épargner la ville même de Nauplie.

Louis-André Gosse allait, lui, se trouver confronté directement avec l'épidémie dans la province de Mégaride. Le premier cas était apparu dans le camp de l'armée grecque du général Démétrius Ypsilanti qui, présupposant, à tort, un cas de typhus, avait fait brûler la maison où le malade était décédé et isoler les deux femmes qui l'avaient soigné. L'une meurt, l'autre s'échappe en emmenant les effets de la première (ces questions de contact par les vêtements semblent primordiales dans la théorie de Gosse); elle gagne la presqu'île de Tycho, région aride où s'étaient réfugiés de nombreux habitants de Thèbes et de Mégare, dans des conditions extrêmement précaires qui favorisaient l'extension de l'épidémie. Gosse est envoyé par Capodistrias sur place en inspection, accompagné d'un aide «actif et intelligent», M.Paul Diamantidys. Ils sont porteurs des médicaments que Gosse juge indispensables. A la fin de son ouvrage, Gosse insère un document qui paraît présenter un intérêt suffisant pour être reproduit: 


\section{Note des principaux médicaments qu'il faut avoir chez soi, dans le moment de la peste. ${ }^{8}$}

$1^{\circ}$ Vomitifs, pour chaque individu de la famille (comme traitement perturbateur).

Vomitif pour homme.

R. Tartre stibié 4 ou 5 grains divisés en 3 prises.

Vomitif pour femme.

R. Tartre stibié 3 ou 4 grains divisés en 3 prises.

Vomitif pour enfants.

Sirop d'ipécacuanha $1 \frac{1}{2}$ once.

$2^{\circ} \quad$ Potasse caustique, soit pierre à cautère (bien sèche et renfermée dans un flacon, à l'abri du contact de l'air, pour appliquer sur les charbons et les bubons).

$3^{\circ}$ Ammoniaque liquide (pour ajouter aux infusions sudorifiques, ou pour préparer l'esprit de Mindererus).

$4^{\circ}$ Sulfate de Quinine (pour prendre mélangé avec un mucilage, dans les intervalles de fièvre).

$5^{\circ}$ Acide sulfurique étendu (pour préparer la limonade sulfurique).

$6^{\circ}$ Camphre (pour préparer l'eau camphrée).

$7^{\circ}$ Feuilles de sauge, fleurs de camomille, de sureau ou autres herbes aromatiques (pour préparer les infusions sudorifiques).

$8^{\circ} \quad$ Essences de térébenthine (pour préparer les onguens stimulans, ou pour appliquer sur les plaies des vésicatoires).

Outre ces médicamens, on fera bien de se pourvoir à la campagne ou dans les établissemens publics,

$9^{\circ}$ De crème de tartre, de magnésie, ou d'huile de ricin (comme laxatifs).

$10^{\circ}$ De moutarde en poudre (pour sinapismes).

$11^{\circ}$ De miel (pour onguent excitant).

$12^{\circ}$ De vinaigre, de citrons ou d'oranges (pour boissons rafraîchissantes ou sudorifiques).

$13^{\circ}$ De mauves, de graine de lin, ou de gomme d'arabie en poudre (pour cataplasmes ou mucilages).

$14^{\circ} \quad D^{\prime}$ esprit de vin rectifié (comme vésicant).

$15^{\circ}$ De chlorure de chaux ou de soude, ou de créosote (comme antigangréneux).

$16^{\circ}$ D'une seringue (pour lavement).

$17^{\circ}$ D'une ou deux lancettes (pour saignées).

$18^{\circ}$ De sangsues, si l'on peut s'en procurer.

$19^{\circ}$ De quelques ventouses.

La plupart de ces remèdes paraissent bien sophistiqués pour être utilisés par de simples particuliers, mais l'un d'entre eux, le fascomilo (soit la sauge sauvage), pousse en abondance dans un terrain caillouteux comme celui de Tycho et Gosse l'a beaucoup utilisé.

Gosse nous a laissé le récit de son équipée ${ }^{9}$ :

«Muni de ces documens, je m'acheminai vers le lieu de la quarantaine, placé à un quart de lieue de la ville. Il me fut difficile de découvrir des malades parmi les familles suspectes, qui 
croyaient encore avoir un intérêt à les cacher; cependant j'en vis 3 ou 4 atteints de véritables charbons et bubons pestilentiels, et en particulier je cauterisai de suite la jambe d'un des soldats du cordon. On m'apprit aussi que la maladie, chez la plupart, ne se communiquait que par un contact très-immédiat, et surtout par la cohabitation sous le même abri et dans le même lit. La misère qui régnait chez ces malheureux contumaces était extrême, et contribuait à aggraver leur position sanitaire. En poursuivant ma course d'inspection, j'arrivai à la presqu'île de Tycho, coteau allongé, rocailleux et entièrement nu, s'avançant dans la mer jusqu'à la distance de demi-lieue, et séparé du continent par une muraille crénelée, d'environ 30 pieds de hauteur, que les réfugiés avaient construite pour se mettre à l'abri des Turcs. A mon entrée dans l'enceinte, le spectacle le plus triste se présenta à mes yeux. D'un côté un hameau presque désert, dont les cabanes renfermaient encore un petit nombre de malades et que parcouraient quelques spectres la bouche couverte d'un mouchoir, dans le but de se préserver de l'influence des miasmes; de l'autre, 120 malheureux qui s'étaient dispersés dans les rochers avec leurs malades, exposés à toutes les intempéries de l'atmosphère, sans secours, sans alimens, sans autre boisson qu'une eau saumâtre infecte. Deux des malades venaient d'expirer, et les chants funèbres de leurs parens, qui se terminaient par des cris déchirans et des convulsions, ajoutaient à la tristesse de cette scène de désolation. Malgré l'ardeur d'un soleil d'été (une température de plus de 33 degrés de Réaumur) et une soif dévorante que je ne pouvais étancher, je parcourus tous les coins et recoins de la presqu'île, et dans l'espace de 5 heures je visitai avec M. Diamantidys 62 malades; j'en recueillis l'observation, et je leur appliquai le traitement le plus urgent, la cautérisation, l'émétique, et le sulfate de Quinine.»

\section{Parmi les mesures originales prises par Gosse à ce moment-là, on peut remarquer la suivante ${ }^{10}$ :}

«Pour seconder le traitement que je viens de tracer, j'eus la précaution, dès mon arrivée à Tycho, de faire construire pour chaque malade une petite cabane de branches tressées, soit une espèce de brancard, élevé de 3 pieds au-dessus du sol, assez long et assez large pour que le malade pût y être commodément couché, et recouvert d'un dôme de verdure partant des côtés des brancards de manière à intercepter les rayons du soleil. Ces cabanes, disposées en dehors du hameau, sur un terrain rocailleux et sur une ligne dirigée de l'est à l'ouest, étaient distantes de six pieds les unes des autres, afin qu'on pût circuler librement autour de chacune, et qu'on eût la facilité d'administrer au malade tous les soins nécessaires, sans être trop immédiatement en contact avec lui. L'ouverture principale, placée du côté du nord, devait répondre aux pieds, et je recommandai qu'on établît de chaque côté, vers la tête, une ouverture fermée à volonté, pour faciliter l'administration des remèdes ou des boissons. Dans un climat comme celui de la Grèce, et dans une saison éminemment chaude et sèche, ces cabanes suffisaient pour mettre le malade à l'abri des rayons du soleil et de la rosée du matin; elles offraient, en outre, l'immense avantage de favoriser un renouvellement continuel de l'air, sans qu'il y eût de courans, circonstance qui, comme je l'ai dit, a non seulement contribué d'une manière puissante au rétablissement des malades, en prévenant le développement des affections typhoïdes et des diarrhées, mais aussi dissipé toute crainte de contagion miasmatique ultérieure et même accéléré l'extinction de la maladie. Faciles à établir, peu coûteuses, et très-commodes pour le service, je ne balance pas à en proposer l'adoption, toutes les fois que la peste régnera dans un climat chaud et sec, surtout lorsqu'il 
s'agira d'y former promptement un hôpital et d'y traiter un plus ou moins grand nombre de malades. - A défaut de branchages, on établira des baraques recouvertes en planches et garnies sur les côtés de tentures en toile, ou bien on se contentera de simples tentes; mais on ne perdra pas de vue, en les établissant, que moins il y aura de malades réunis dans une même enceinte, plus on aura de chances de réussite dans l'application du traitement.»

Ces principes de quarantaine sont les mêmes appliqués en «dur» dans le lazaret construit à Egine selon les plans de M. Groppius, consul d'Autriche en Grèce, et dont Gosse fournit le schéma à la première page de son ouvrage (voir fig.2). Gosse en a fait un commentaire détaillé, presque utopique. ${ }^{11}$

«Adossé contre le promontoire de la colonne qui l'abrite au nord, cet établissement est légèrement incliné vers le sud. Sur le devant est une place publique et un quai s'étend le long de l'ancien port. Il consiste en un édifice central A, dont le rez-de-chaussée sert de bureau et d'archives et le premier étage d'habitation pour le directeur. Aux quatre angles de cette maison sont placés des cabinets d'inspection. - De chaque côté sont les portes grillées, qui servent d'entrée dans le lazaret. - En dedans, règne une cour centrale semi-circulaire B, qui communique directement avec l'intérieur des cours de contumaces CC, par le moyen de deux grilles FF, placées à dix pieds de distance l'une de l'autre, et percées de portes qui ne se

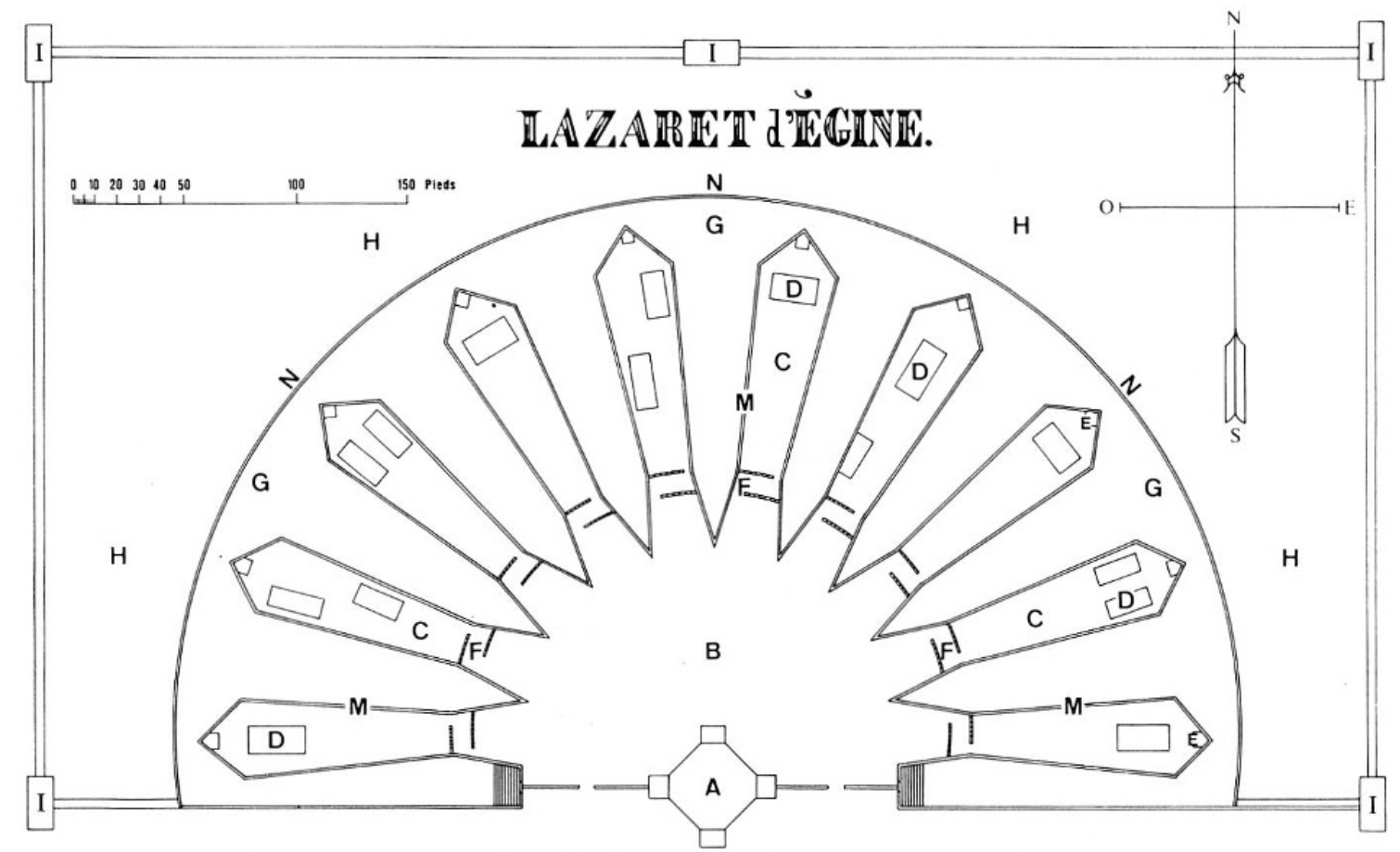
A.- Administration.
B.- Cour centrale.
C.- Enceintes
isolées avec Pavillons d'habitation pour les personnes en quarantaine. D.. Pavillons.
E. - Latrines.
F. - Espace entre les grilles servant de Parloirs
G. Enceinte basse avec un mur de clôture. H. Cimetière entouré d'un fossé.
M.M. _- Premier mur d'enceinte. N.N.N._ Second mur d'enceinte.
I. - Vedettes.

Fig. 2 Le lazaret d'Egine pour les pestiférés, en 1828, d'après L.-A.Gosse 
correspondent pas directement. Ces doubles grilles servent de parloir pour les contumaces, et pour le public qui, à certaines heures, est admis dans la cour centrale. - Les cours de contumaces, sur le même niveau que la cour centrale, sont au nombre de dix, et disposées en éventail. Dans ces cours sont construites les habitations des contumaces DD et les cuisines; elles ne consistent qu'en un rez-de-chaussée, et le nombre en varie de 1 à 4 dans chaque cour, afin d'isoler, suivant le besoin, les diverses familles qui entrent le même jour en quarantaine. A l'extrémité de chaque cour sont placées les latrines EE, qui communiquent avec l'extérieur. - Les cours de contumaces sont environnées d'un premier mur MM, qui forme des saillies en éperon dans la cour centrale, afin d'isoler, le plus complètement possible, les parloirs des diverses catégories de contumaces. La hauteur de ce mur est de 10 pieds en dedans, mais elle est presque du double au dehors, qui correspond à une cour d'enceinte GG dont le niveau est inférieur à celui des cours de contumaces. Cette dernière cour sert d'entrepôt pour les marchandises; elle sert aussi à leur purification et à leur aération, et les latrines s'y vident. Un second mur d'enceinte NN assez élevé l'environne; il vient rejoindre de chaque côté l'entrée du lazaret, et en arrière il forme un vaste demi-cercle. Deux escaliers KK, munis de portes, placées près des entrées, conduisent dans la cour d'enceinte depuis l'extérieur et depuis la cour centrale. Enfin, en dehors du second mur, on a disposé un carré de terrain $\mathrm{HH}$, pour servir de cimetière aux contumaces qui viendraient à succomber. Ce cimetière est ceint d'un fossé, et de petits corps-de-garde IIII sont placés de distance en distance pour y loger des vedettes.

\section{Les avantages du lazaret d'Egine sont:}

$1^{\circ}$ Une inspection facile, économique et complète, depuis l'édifice central où réside le directeur.

$2^{\circ}$ La séparation complète des diverses catégories de contumaces.

$3^{\circ}$ L'inviolabilité du lazaret, assurée autant que possible par une double enceinte de murs et par l'établissement de corps-de-garde extérieurs, sans parler des avantages d'une surveillance de tous le momens.

$4^{\circ}$ L'aération de toutes les parties de l'établissement, sans nuire à sa sûreté.

$5^{\circ}$ La facilité de purifier les effets, sans embarrasser le service de l'intérieur.

$6^{\circ}$ La facilité des communications à distance entre les contumaces et la population en libre pratique, à l'aide des nombreux parloirs, sans mélange des diverses catégories de contumaces, et sans crainte d'infractions aux lois de la quarantaine.

$7^{\circ}$ La facilité d'entretenir la propreté et la sécheresse dans l'intérieur des cours de contumaces, à l'aide des latrines donnant sur la cour d'enceinte, et de la différence de niveau de ces cours.

$8^{\circ}$ La facilité d'enterrer les morts du lazaret, sans communication avec l'extérieur et sans nuire à la salubrité de l'établissement.

On pourrait reprocher à ce petit lazaret l'absence d'une double enceinte extérieure du côté de la place publique; le défaut d'étendue de la cour d'enceinte; l'absence de communication directe du lazaret à la mer, et d'un port uniquement destiné à la quarantaine des bâtimens et de leurs équipages; la disposition vicieuse de quelques-unes des habitations des contumaces, qui s'oppose à l'inspection facile d'une portion des cours; le manque de magasins vastes pour les marchandises; enfin le manque d'eau dans chacune des cours. Mais ces défauts, qui 
tiennent en partie à la nature des localités et à la destination restreinte de ce lazaret, sont si minimes, en comparaison des avantages énumérés, qu'ils ne sauraient entrer en ligne de compte.»

Gosse charge Diamantidys d'appliquer à Tycho le traitement qu'il a préconisé et rentre à Poros, où il fait son rapport à Capodistrias. L'île échappe à la contagion, grâce aux mesures de quarantaine appliquées avec rigueur par la commission sanitaire dont il fait partie, mais aussi grâce au fait, que Gosse souligne sans pouvoir l'expliquer, qu'aucune des régions touchées chroniquement par les fièvres pernicieuses (la malaria) - c'est le cas de Poros - n'a été contaminée par la peste. Mais peu de temps après son retour, par une chaleur écrasante, éclate une épidémie de ces fièvres parmi les quelque deux mille réfugiés athéniens parqués dans la région marécageuse en face de Poros et qui s'abritent dans de petites huttes en feuillage où, pour les soigner, Gosse est obligé de pénétrer en rampant. Il a désormais tous les jours, en plus de ces visites sur place, une consultation gratuite dans sa chambre où il distribue les médicaments amenés de Genève, principalement le sulfate de quinine. Sur huit cents malades qu'il a traités, il n'en meurt qu'un petit nombre, mais après six semaines de cette galère, il tombe à son tour malade. Il est à plusieurs reprises au bord de la mort. Il est soigné avec compétence à l'hôpital américain fondé par le $\mathrm{D}^{\mathrm{r}}$ Howe de Boston et le Dr Rush de Philadelphie grâce à la générosité des philhellènes américains, mais il expérimente sur lui-même des méthodes violentes et originales ${ }^{12}$ :

«Le soir de mon entrée [à l'hôpital], je m'aperçus des prodromes du second accès; il était onze heures du soir. N'ayant reçu aucun traitement pour prévenir l'attaque, je sentis que je devais y succomber, par la violence des accidents, mais tout à coup il me vint une espèce de résolution instinctive, de sortir de l'hôpital et de me faire promener sur la mer.

Le Dr Rush crut que cette idée était le résultat du délire et pour me calmer, il m'assura que l'on ne trouverait point de matelots à cette heure pour diriger l'embarcation. Alors mon exaltation augmenta; je lui dis de s'adresser au gouverneur de la ville, on me répondit qu'il était absent, puis à Capodistrias lui-même pour obtenir des matelots, enfin, voyant mon obstination, il me procura un bateau et m'y transporta presque mourant, il m'y accompagna avec une boisson rafraîchissante. Il régnait une brise légère et fraîche qui diminua peu à peu mon agitation et après plusieurs bordées, je le priai de me mettre à terre auprès d'un couvent où je savais qu'il y avait une source très fraîche et où j'espérais calmer la soif intense que j'éprouvais. Débarqué sur la grève, le danger de l'accès avait diminué et je pus m'endormir pendant une demi-heure, ce que je n'avais pas fait depuis quinze jours. Je me fis ensuite transporter dans le couvent situé sur la hauteur et j'employai cette eau fraîche pour arroser ma chambre, plancher et parois. [Il précise dans son Journal ${ }^{13}$ : con m'entra dans une petite chambre dont on ferma portes et fenêtres et où je restai tout nu, employant un 
prisonnier turc à inonder incessament ma chambre et moi-même d'eau fraîche et m'occupant sans cesse à me gargariser sans en jamais avaler et en la rejetant.»] L'accès de fièvre passé, je pus alors prendre de la quinine et pus sortir au bout de quelques jours, mais d'une faiblesse telle que je pouvais à peine marcher.»

S'ensuit une période de convalescence fort agréable à Syra et surtout dans la maison de campagne du comte Frangopoulos à Naxos (que Gobineau allait décrire dans une nouvelle célèbre). Mais il souffrira jusqu'à son retour en Suisse d'accès périodiques de fièvre. De retour à Poros, il travaille à liquider ses comptes de la flotte et - le moral n'y est plus - il montre une impatience de plus en plus grande de rentrer en Suisse.

Mais, avant ce retour qui aura lieu en mai 1829, il n'hésite pas néanmoins à accepter une nouvelle mission d'inspection, dans le troisième foyer de l'épidémie, la province d'Achaïe, intéressé par la forme que prend la maladie dans un climat froid ${ }^{14}$. L'épidémie, qui avait sévi dans cette région en été, semblait avoir disparu, puis avait subitement réapparu dans les mêmes villages dès la fin novembre. Parti d'Egine le jour de Noël, Gosse traverse l'isthme de Corinthe et continue par mer en direction de Patras. Grelottant de fièvre, il est accueilli au château de Lépante par les officiers français de l'armée du général Maison. De là, il s'achemine vers les montagnes de Kalavryta où il est obligé de passer la nuit à la belle étoile; le lendemain, il traverse des ravins dangereux le long de sentiers presque impraticables et par une «neige battante», et le 4 janvier, il atteint enfin le village de Vizoka, après avoir été sur le point de périr de froid et de fièvre dans les neiges qui couvraient les cols. Il y examine un certain nombre de malades, donne des instructions précises à l'hygionome de la région, cherche à procurer à ceux qui sont en quarantaine les secours alimentaires nécessaires. Selon les conseils donnés à son retour dans son rapport, Capodistrias enverra un médecin à Kalavryta pour surveiller l'exécution des mesures sanitaires; mais la maladie disparaît d'elle-même à la fin de l'hiver.

Dans la conclusion de la partie historique de son ouvrage, Gosse, tout en insistant sur la nécessité de mesures préventives, constatait que sur 1113 malades recensés, 783 étaient morts et 330 s'en étaient sortis. La maladie s'était présentée de manière menaçante; mais étant étrangère au sol, au climat de la Grèce, trouvant des populations rares et dispersées, elle s'était évanouie dès le printemps 1829, sans laisser de traces durables.

Faute de place et de compétence, nous n'avons pas présenté dans cette étude le point de vue de Gosse concernant les causes prédisposantes et déterminantes de la maladie, les symptômes, le diagnostic, le prognostic, le 
traitement, les mesures préservatives. On remarque constamment dans ces chapitres son souci d'objectivité, lorsqu'il expose les méthodes qui ne sont pas les siennes; par exemple, sur l'opportunité de la saignée notamment, et il est fait souvent allusion à l'école de Broussais dont il ne partage probablement pas toutes les vues.

Ce qui ressort en fin de compte de sa Relation, de son Journal, de son Séjour, de ses lettres, c'est sa probité absolue, son désintéressement, son courage et son dévouement sans limite à la cause qu'il défend.

\section{Notes}

1 Bouvier-Bron Michelle, Le séjour en Grèce de Louis-André Gosse (1827-1829), mémoire de licence présenté à Genève, en mars 1958 (dactylographié), p. 38-39.

2 Gosse Louis André, Relation de la peste qui a régné en Grèce en 1827 et 1828, contenant des vues nouvelles sur la marche et le traitement de cette maladie, Paris, 1838.

3 Bouvier-Bron, op. cit., p. 46.

4 ibidem, p. 55.

5 ibidem, p. 66-67.

6 ibidem, p. 77.

7 Gosse, Relation ..., p. 16-17.

8 ibidem, p. 140-141.

9 ibidem, p. 28-29.

10 ibidem, p. 134-135.

11 ibidem, p. 183-186.

12 Bouvier-Bron, op. cit., p. 80-81.

13 Bibliothèque publique et universitaire, ms 2667, dossier 10, Fragment de Journal (copié ou dicté, d'une autre main), 22 juillet 1828.

14 Une carte détaillée des villages touchés par l'épidémie figure dans l'ouvrage de C. A. Vacalopoulos, Relations entre Grecs et philhellènes suisses pendant la guerre d'Indépendance de 1821 (en grec), Thessalonique, 1975, p. 171. 


\section{Summary}

The medical mission of Louis-André Gosse in Greece (1827-1829)

Louis-André Gosse (1791-1873), a liberal-minded Geneva physician, went to Greece in 1827 in order to work, for a couple of years, for the Greek State, which had won its independence just a few years ago. When, in 1828, an Egyptian army brought plague back to Greece, Gosse took an active part in the fight against the epidemic. The present author gives a detailed account of Gosse's relation of his experience including treatment and preventive measures.

\section{Zusammenfassung}

\section{Die ärztliche Mission von Louis-André Gosse in Griechenland (1827-1829)}

Louis-André Gosse (1791-1873), ein Genfer Arzt liberaler Geistesrichtung, war durch das Elend, das in Griechenland während des 1821 begonnenen Freiheitskampfes herrschte, stark beeindruckt; er verliess seine Praxis, um sich zeitweilig in den Dienst des jungen Staates zu stellen. Während seines Aufenthalts 1827-1829 betreute er vorerst als Kommissar der Flotte und Steuereinnehmer des Archipels wichtige Aufgaben in der Verwaltung und nahm dann auf Verlangen des Präsidenten Johannes Kapodistrias an der Bekämpfung der im Frühling 1828 ausgebrochenen Pestepidemie regen Anteil. Die Krankheit, seit langer Zeit aus Griechenland verschwunden, wurde durch das ägyptische Heer zurückgebracht und verbreitete sich über die Inseln Aigina, Hydra und Spetses in den Provinzen Argolis, Megaris und Achaia. Dank rascher, energischer Massnahmen konnte der Seuche Einhalt getan werden, doch forderte sie jedoch an die 800 Menschenopfer. Der Arzt L.-A.Gosse leistete in der Megaris im Juni 1828, danach in Achaia im Januar 1829, vorbildliche Feldarbeit und verfasste später einen eingehenden Bericht über seine die Pest betreffenden Erfahrungen und Erkenntnisse, die angewandte Behandlung und die unerlässlichen Vorbeugungsmassnahmen.

Mme Michelle Bouvier-Bron

9, Rue de l'Encyclopédie

CH-1201 Genève 